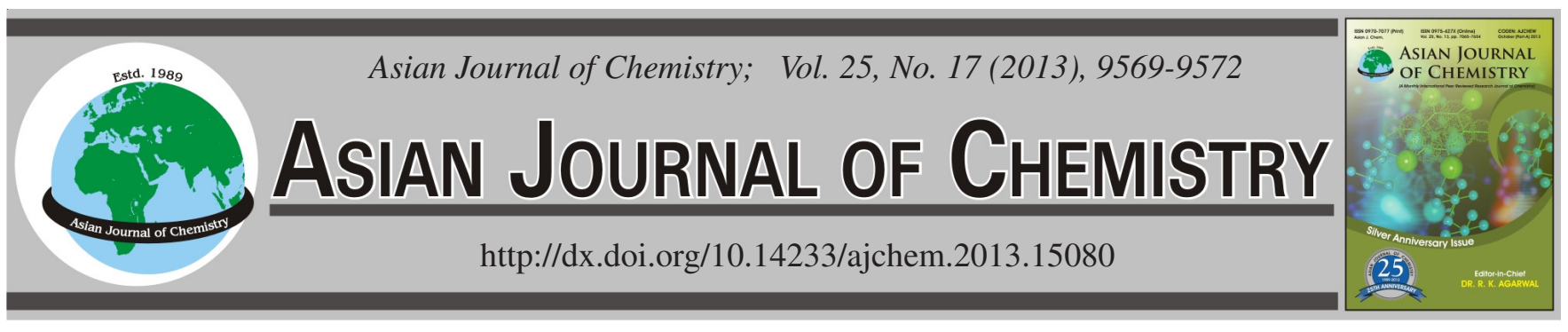

\title{
Green Syntheses of N-Alkyl-2-styrylbenzimidazoles
}

\author{
T. AshoK Kumar*, B. Rama Devi and P.K. Dubey
}

Department of Chemistry, Jawaharlal Nehru Technological University Hyderabad, College of Engineering, Kukatpally, Hyderabad-500 085, India

*Corresponding author: E-mail: ashok.jntu@gmail.com

(Received: 2 January 2013;

Accepted: 4 October 2013)

AJC-14231

\begin{abstract}
Simple and green methodologies for the syntheses of 2-styrylbenzimidazoles (3a-c) and its N-alkyl derivatives (7a-i) have been developed. $o$-Phenylenediamine (1) was condensed with cinnamic acids (2a-c) resulting in 2-styrylbenzimidazoles (3a-c) using glycerol as a green and efficient solvent. 3 were also prepared alternatively by the condensation of 2-methylbenzimidazole (4) with benzaldehydes (5a-c) using glycerol as solvent. 2-Styrylbenzimidazoles (3a-c) and 2-methylbenzimidazole (4) were alkylated independently to obtain $\mathrm{N}$-alkyl2-styrylbenzimidazole (7a-i) and $\mathrm{N}$-alkyl-2-methylbenzimidazole (6a-c), respectively using $\mathrm{DMS} / \mathrm{DES} / \mathrm{PhCH}_{2} \mathrm{Cl}$ applying green methods such as simple physical grinding of reactants in solid phase, treating reactants in PEG-600 as a solvent in solution phase and using microwave irradiation of reactants respectively. Compounds 7a-i could also be prepared, alternatively, by heating $\mathbf{6 a - c}$ with $\mathbf{5 a - c}$ in glycerol at $180^{\circ} \mathrm{C}$ for 3-4 h.
\end{abstract}

Key Words: 2-Styrylbenzimidazoles, Glycerol, Alkylations, Physical grinding, Microwave irradiation, PEG-600.

\section{INTRODUCTION}

The development of green methods for syntheses of target compounds from easily available starting materials is a major challenge to the present day organic chemist. In this category, glycerol mediated ${ }^{1-3}$ reactions are very useful for the synthesis of important heterocycles such as benzimidazoles which are known to possess a range of biological activities such as antihypertensive ${ }^{4}$, antiviral ${ }^{4}$ and anticancer types ${ }^{5}$. Compounds that contain benzimidazole skeleton exhibit significant activity against several viruses such as $\mathrm{HIV}^{6}$, herpes simplex virus Type-1 (HSV-1) ${ }^{7}$, influenza ${ }^{8}$ and human cytomegalovirus $(\mathrm{HCMV})^{6}$

Although many methods are available for the synthesis of 2-substituted benzimidazoles ${ }^{9-16}$, not much work seems to have been done to develop green synthetic methods to prepare these types of compounds. Among the most notable green methods used in synthesis, solid phase synthesis ${ }^{17,18}$, solution phase synthesis using green solvents ${ }^{19}$ and microwave techniques $^{20}$ are very important. In continuation of our earlier work on synthesis of 2-styrylbenzimidazoles ${ }^{21-24}$, we now wish to report our studies on preparation and $\mathrm{N}$-alkylation of 2-styrylbenzimidazoles using green methodologies.

\section{EXPERIMENTAL}

All the reagents used in this work were obtained from commercial suppliers. Solvents were freshly distilled before being used. Melting points were determined using a Buchi Melting Point B-545 apparatus and are uncorrected. TLC analyses were done on glass plates coated with silica gel GF254 and spotting was done using iodine/UV lamp. IR spectra were recorded on a Perkin-Elmer model 446 instrument in $\mathrm{KBr}$ phase. ${ }^{1} \mathrm{H}$ NMR were recorded in $\mathrm{CDCl}_{3} / \mathrm{DMSO}$ using $400 \mathrm{MHz}$ Varian Gemini spectrometer and mass spectra were recorded on LC-MS spectrometer, model HP-5989A.

Preparation of 2-styrylbenzimidazoles (3a-c) from $o$ phenylenediamine (1): An intimate mixture of $\mathbf{1}$ (1.08 g, $10 \mathrm{mM})$, cinnamic acids (2a-c) $(10 \mathrm{mM})$ and glycerol (10 mL) was heated at $170-180^{\circ} \mathrm{C}$ for $1 \mathrm{~h}$. At the end of this period, the reaction mixture was poured into ice cold water. The separated solid was filtered, washed with water and dried. The crude products were recrystallized from suitable solvent to get pure 3a-c.

Alternative preparation of 2-styrylbenzimidazoles (3ac) from 2-methylbenzimidazole (4): A mixture of 4 (1.32 g, $10 \mathrm{mM})$, aromatic aldehydes $(\mathbf{5 a}-\mathbf{c})(10 \mathrm{mM})$ and glycerol $(10 \mathrm{~mL})$ was heated at $170-180^{\circ} \mathrm{C}$ for $3 \mathrm{~h}$ in an oil bath. At the end of this period, the reaction mixture was cooled to room temperature, dissolved in isopropanol $(25 \mathrm{~mL})$ and treated with a solution of oxalic acid $(1.5 \mathrm{~g})$ in isopropanol $(10 \mathrm{~mL})$. Each of the oxalate salts of (3a-c) obtained were filtered and neutralized, independently, with aqueous $\mathrm{NH}_{3}$ to $\mathrm{pH}$ of 8-10. The products were filtered, washed with water, dried and recrystallized using suitable solvent to obtain 3a-c. 
Preparation of $\mathrm{N}$-alkyl-2-styrylbenzimidazoles (7a-i) from $\mathrm{N}$-alkyl-2-methylbenzimidazoles [6(a-c)]: The procedure is same as mentioned above for the synthesis of 3a-c from 4 .

Preparation of $\mathrm{N}$-alkyl-2-styrylbenzimidazoles (7a-i) \& N-alkyl-2-methylbenzimidazoles [6(a-c)]: In solid phase (Physical grinding method). A mixture of 3a-c or 4 (10 mM), $\mathrm{K}_{2} \mathrm{CO}_{3}(20 \mathrm{mM})$, tetrabutylammonium bromide $(1 \mathrm{mM})$ and alkylating agent $(10 \mathrm{mM})$ were ground together independently for about 10-15 min in a mortar with a pestle at room temperature to obtain a homogeneous mixture. The latter was then treated with ice-cold water $(30-40 \mathrm{~mL})$. The separated solid was filtered, washed with water $(2 \times 10 \mathrm{~mL})$ and dried to obtain crude $6 \mathbf{a}-\mathbf{c}$ or $\mathbf{7 a - i}$ respectively, which were recrystallized from a suitable solvent to obtain pure $\mathbf{7 a - i}$ or $\mathbf{6 a - c}$ respectively. For yields (Table-2).

In solution phase (In PEG-600): A mixture of 3a-c or 4 $(10 \mathrm{mM})$, alkylating agent $(10 \mathrm{mM})$ and PEG-600 $(20 \mathrm{~mL})$ were taken to heat at $100{ }^{\circ} \mathrm{C}$ on water bath for $2 \mathrm{~h}$. At the end of this period, the reaction mixture was poured into ice-cold water. The separated solid was filtered, washed with water and dried. The crude products were recrystallized from suitable solvent to obtain pure 7a-i or $\mathbf{6 a - c}$ respectively. For yields (Table-3).

Under microwave irradiation condition: A mixture of 3a-c or 4 (10 mM) dissolved in PEG-600 (10 mL) and alkylating agent $(10 \mathrm{mM})$ was added and taken in a $10 \mathrm{~mL}$ CEMreaction tube sealed by rubber stopper and subjected to microwave irradiation for $5 \mathrm{~min}$ at $130{ }^{\circ} \mathrm{C}$ in the commercial micro-wave reactor. After that, the tube was cooled and the completion of reaction was checked by TLC then poured into ice-cold water. The separated solid was filtered, washed with water and dried. The crude product was recrystallized from a suitable solvent to obtain pure $\mathbf{7 a - i}$ or $\mathbf{6 a - c}$ respectively. For yields (Table-4).

Physical and spectral data of the obtained compounds are given below.

1-Methyl-2-styryl-1H-benzimidazole (7a) (Table-1, entry 4): Yield (1.6g, $70 \%$ ), m.p. $114-115^{\circ} \mathrm{C}$ (Lit. m.p. ${ }^{24}$ 112-114), IR (KBr, $\left.v_{\max }, \mathrm{cm}^{-1}\right): 3010(-\mathrm{CH}=\mathrm{CH}), 1624(\mathrm{C}=\mathrm{N})$, ${ }^{1} \mathrm{H}$ NMR (400 MHz, DMSO- $\left.d_{6}\right): \delta 3.6(3 \mathrm{H}, \mathrm{s},-\mathrm{N}-\mathrm{CH}), \delta 6.9-$ $7.0(2 \mathrm{H}, \mathrm{dd}$, trans $-\mathrm{CH}=\mathrm{CH}), \delta$ 7.1-7.3 (5H, m, aromatic) $\delta$ 7.2-7.7 (4H, m, aromatic); MS: $m / z 249.13\left(\mathrm{M}^{+}\right)$. Anal. calcd. (\%) for $\mathrm{C}_{16} \mathrm{H}_{14} \mathrm{~N}_{2}$ : C, 82.02; H, 6.02; N, 11.96; Found C, 82.12; $\mathrm{H}, 6.10 ; \mathrm{N}, 11.99$.

1-Ethyl-2-styryl-1H-benzimidazole (7b) (Table-1, entry 5): Yield (1.6 g, $68 \%$ ), m.p. $158-160{ }^{\circ} \mathrm{C}$ (Lit. m.p. ${ }^{24} 160-$ $\left.161{ }^{\circ} \mathrm{C}\right)$, IR $\left(\mathrm{KBr}, v_{\max }, \mathrm{cm}^{-1}\right): 3010(-\mathrm{CH}=\mathrm{CH}), 1625(\mathrm{C}=\mathrm{N})$, ${ }^{1} \mathrm{H}$ NMR (400 MHz, DMSO- $\left.d_{6}\right)$ : $\delta 1.5(3 \mathrm{H}, \mathrm{t}, \mathrm{N}-\mathrm{C}-\mathrm{CH}), \delta 3.7-$ $3.8(2 \mathrm{H}, \mathrm{q}, \mathrm{N}-\mathrm{CH}), \delta 6.9-7.0(2 \mathrm{H}$, dd, trans-CH=CH), $\delta 7.1-$ $7.3(5 \mathrm{H}, \mathrm{m}$, aromatic), $\delta$ 7.3-7.7 (4H, m, aromatic) ; MS: $\mathrm{m} / \mathrm{z}$ $249.13\left(\mathrm{M}^{+}\right)$. Anal. calcd. (\%) for $\mathrm{C}_{17} \mathrm{H}_{16} \mathrm{~N}_{2}$ : C, 82.22; H, 6.49; N, 11.28; Found C, 82.42; H, 6.56; N, 11.30.

1-Benzyl-2-styryl-1H-benzimidazole (7c) (Table-1, entry 6): Yield (2.1 g, $70 \%$ ), m.p. $120-121{ }^{\circ} \mathrm{C}$ (Lit. m.p. 120 $\left.{ }^{\circ} \mathrm{C}\right)$, IR $\left(\mathrm{KBr}, \mathrm{v}_{\max }, \mathrm{cm}^{-1}\right): 3020(-\mathrm{CH}=\mathrm{CH}), 1624(\mathrm{C}=\mathrm{N}),{ }^{1} \mathrm{H}$ NMR (400 MHz, DMSO- $\left.d_{6}\right)$ : $\delta$ 4.9-5.0 (2H, s, N-CH), $\delta 6.9-$ $7.0(2 \mathrm{H}, \mathrm{dd}$, trans $-\mathrm{CH}=\mathrm{CH}), \delta$ 7.0-7.4 $(5 \mathrm{H}, \mathrm{m}$, aromatic $), \delta$ 7.1-7.3 (5H, m, aromatic), $\delta$ 7.3-7.8 (4H, m, aromatic), MS: $m / z 311.15\left(\mathrm{M}^{+}\right)$. Anal. calcd. (\%) for $\mathrm{C}_{22} \mathrm{H}_{18} \mathrm{~N}_{2}: \mathrm{C}, 85.13 ; \mathrm{H}$, 5.85; N, 9.03; Found C, 85.16; H, 5.92; N, 9.79.
1-Methyl-2-(2-p-tolyl-vinyl)-1H-benzimidazole (7d) (Table-1, entry 7): Yield (1.8 g, $72 \%$ ), m.p. $129-130{ }^{\circ} \mathrm{C}$ (Lit. m.p. $\left.{ }^{24} 128-130\right)$, IR (KBr, $\left.v_{\max }, \mathrm{cm}^{-1}\right): 3010(-\mathrm{CH}=\mathrm{CH})$, $1625(\mathrm{C}=\mathrm{N}),{ }^{1} \mathrm{H}$ NMR $\left(400 \mathrm{MHz}, \mathrm{DMSO}-d_{6}\right): \delta 2.3(3 \mathrm{H}, \mathrm{s}$, $-\mathrm{CH}), \delta 3.6(3 \mathrm{H}, \mathrm{s}, \mathrm{N}-\mathrm{CH}), \delta$ 6.9-7.0 $(2 \mathrm{H}, \mathrm{dd}$, trans $-\mathrm{CH}=\mathrm{CH})$, d 7.0-7.2 (4H, q, aromatic), $\delta$ 7.3-7.7 (4H, m, aromatic), MS: $m / z 249.13\left(\mathbf{M}^{+}\right)$. Anal. calcd. (\%) for $\mathrm{C}_{17} \mathrm{H}_{16} \mathrm{~N}_{2}$ : C, 82.22; $\mathrm{H}$, 6.49; N, 11.28; Found C, 82.30; H, 6.55; N, 11.30.

1-Ethyl-2-(2-p-tolyl-vinyl)-1H-benzimidazole (7e) (Table-1, entry 8): Yield (1.8 g, $70 \%$ ), m.p. $108-110{ }^{\circ} \mathrm{C}$, IR $\left(\mathrm{KBr}, \mathrm{v}_{\max }, \mathrm{cm}^{-1}\right): 3010(-\mathrm{CH}=\mathrm{CH}), 1625(\mathrm{C}=\mathrm{N}),{ }^{1} \mathrm{H}$ NMR $(400$ $\left.\mathrm{MHz}, \mathrm{DMSO}-d_{6}\right): \delta 1.5(3 \mathrm{H}, \mathrm{t}, \mathrm{N}-\mathrm{C}-\mathrm{CH}), \delta 2.3-2.5(3 \mathrm{H}, \mathrm{s}$, $-\mathrm{CH}), \delta$ 3.7-3.8 (2H, q, N-CH), $\delta$ 6.9-7.0 (2H, dd, trans$\mathrm{CH}=\mathrm{CH}), \delta$ 7.0-7.2 (4H, q, aromatic) $\delta 7.3-7.7$ (4H, m, aromatic); MS: $m / z$ 263. 35(M $\left.\mathrm{M}^{+}\right)$. Anal. calcd. (\%) for $\mathrm{C}_{18} \mathrm{H}_{18} \mathrm{~N}_{2}$ : C, 82.41; H, 6.92; N, 10.68; Found C, 82.44; H, 6.98; N, 10.80 .

N-Benzyl-2-(2-p-tolyl-vinyl)-1H-benzimidazole (7f) (Table-1, entry 9): Yield (2.1 g, $65 \%$ ), m.p. $210-212{ }^{\circ} \mathrm{C}$, IR $\left(\mathrm{KBr}, \mathrm{v}_{\max }, \mathrm{cm}^{-1}\right): 3020(-\mathrm{CH}=\mathrm{CH}), 1624(\mathrm{C}=\mathrm{N}),{ }^{1} \mathrm{H}$ NMR $(400$ $\left.\mathrm{MHz}, \mathrm{DMSO}-d_{6}\right): \delta 2.3-2.5(3 \mathrm{H}, \mathrm{s},-\mathrm{CH}), \delta$ 4.9-5.0 $(2 \mathrm{H}, \mathrm{s}$, $\mathrm{N}-\mathrm{CH}), \delta$ 6.9-7.0 (2H, dd, trans-CH=CH), $\delta$ 7.0-7.2 (9H, m, aromatic), $\delta$ 7.3-7.7 (4H, m, aromatic) ; MS: $\mathrm{m} / \mathrm{z} 325.12\left(\mathrm{M}^{+}\right)$. Anal. calcd. (\%) for $\mathrm{C}_{23} \mathrm{H}_{20} \mathrm{~N}_{2}$ : C, 85.15; H, 6.21; N, 8.63; Found C, 85.24; H, 6.28; N, 8.72.

2-[2-(4-Chloro-phenyl)-vinyl]-1-methyl-1H-benzimidazole (7g) (Table-1, entry 10): Yield (1.9 g, 73 \%), m.p. 143-145 ${ }^{\circ} \mathrm{C}$ (Lit. m.p. $\left.{ }^{24} 142-143{ }^{\circ} \mathrm{C}\right), \mathrm{IR}\left(\mathrm{KBr}, \nu_{\max }, \mathrm{cm}^{-1}\right)$ : 3010 $(-\mathrm{CH}=\mathrm{CH}), 1625(\mathrm{C}=\mathrm{N}),{ }^{1} \mathrm{H}$ NMR $\left(400 \mathrm{MHz}, \mathrm{DMSO}-d_{6}\right): \delta$ $3.6(3 \mathrm{H}, \mathrm{s}, \mathrm{N}-\mathrm{CH}), \delta$ 6.9-7.0 (dd, $2 \mathrm{H}$, trans $-\mathrm{CH}=\mathrm{CH}), \delta 7.3-$ 7.8 (8H, m, aromatic); MS: $m / z 269\left(\mathrm{M}^{+}\right)$. Anal. calcd. (\%) for $\mathrm{C}_{16} \mathrm{H}_{13} \mathrm{~N}_{2} \mathrm{Cl}$ : C, 71.51; H, 4.88; Cl, 13.19; N, 10.42; Found C, 71.60; H, 4.92; Cl, 13.30; N, 10.55 .

2-[2-(4-Chloro-phenyl)-vinyl]-1-ethyl-1H-benzimidazole (7h) (Table-1, entry 11): Yield (1.9 g, 70 \%), m.p. 136$138^{\circ} \mathrm{C}$, IR $\left(\mathrm{KBr}, v_{\max }, \mathrm{cm}^{-1}\right): 3010(-\mathrm{CH}=\mathrm{CH}), 1624(\mathrm{C}=\mathrm{N})$, ${ }^{1} \mathrm{H}$ NMR $\left(400 \mathrm{MHz}, \mathrm{DMSO}-d_{6}\right): \delta 1.5(3 \mathrm{H}, \mathrm{t}, \mathrm{N}-\mathrm{C}-\mathrm{CH}), \delta 3.7-$ $3.8(2 \mathrm{H}, \mathrm{q}, \mathrm{N}-\mathrm{CH}), \delta 6.9-7.0(2 \mathrm{H}$, dd, trans $-\mathrm{CH}=\mathrm{CH}), \delta 7.2-$ $7.8\left(8 \mathrm{H}, \mathrm{m}\right.$, aromatic); MS: $m / z 283.7\left(\mathrm{M}^{+}\right)$. Anal. calcd. (\%) for $\mathrm{C}_{17} \mathrm{H}_{15} \mathrm{~N}_{2} \mathrm{Cl}$ : C, 72.21; H, 5.35; Cl, 12.54; N, 9.91; Found C, 72.30; H, 5.42; N.9.99.

N-Benzyl-2-[(E)-2-(4-chlorophenyl)ethenyl]-1H-1,3benzimidazole (7i). (Table-1, entry 12): Yield (2.4 g, $72 \%)$, m.p. $>230{ }^{\circ} \mathrm{C}$, IR $\left(\mathrm{KBr}, v_{\max }, \mathrm{cm}^{-1}\right): 3020(-\mathrm{CH}=\mathrm{CH}), 1625$ $(\mathrm{C}=\mathrm{N}),{ }^{1} \mathrm{H}$ NMR $\left(400 \mathrm{MHz}, \mathrm{DMSO}-d_{6}\right): \delta 4.9-5.0(2 \mathrm{H}, \mathrm{s}, \mathrm{N}-$ $\mathrm{CH}), \delta$ 6.9-7.0 (2H, dd, trans- $\mathrm{CH}=\mathrm{CH}), \mathrm{d} 7.0-7.2(5 \mathrm{H}, \mathrm{m}$, aromatic), $\delta$ 7.3-7.8 (8H, m, aromatic); $\mathrm{MS}: \mathrm{m} / z 345.42\left(\mathrm{M}^{+}\right)$. Anal. calcd. (\%) for $\mathrm{C}_{22} \mathrm{H}_{17} \mathrm{~N}_{2} \mathrm{Cl}$ : C, 76.63; H, 4.97; Cl, 10.28; N, 8.12; Found C, 76.70; H, 4.99; Cl, 10.35; N, 8.26.

\section{RESULTS AND DISCUSSION}

Reaction of $o$-phenylenediamine (1) with cinnamic acids (2a-c) in glycerol at $180^{\circ} \mathrm{C}$ resulted in 2-styrylbenzimidazoles (3a-c) (i.e., 3a, $\left.\mathrm{Ar}=\mathrm{C}_{6} \mathrm{H}_{5}\right),\left(\mathbf{3 b}, \mathrm{Ar}=\mathrm{C}_{6} \mathrm{H}_{4}-p-\mathrm{Cl}\right)$ and $(\mathbf{3 c}, \mathrm{Ar}=$ $\mathrm{C}_{6} \mathrm{H}_{4}-p-\mathrm{CH}_{3}$ ) in good yields (Table-1) and the products were identical with the ones reported in earlier methods ${ }^{25,26}$ in all respects (m.p. m.m.p and co-TLC analysis). 2-Methylbenzimidazole (4), which was prepared by Philip's condensation ${ }^{27}$, condensation of 1 with acetic acid using $4 \mathrm{~N} \mathrm{HCl}$, on reacting with substituted benzaldehydes (5a-c) (i.e., 5a, $\mathrm{Ar}=\mathrm{C}_{6} \mathrm{H}_{5}$ ), 
TABLE-1

SYNTHESIS OF COMPOUNDS 3a-c AND 7a-i FROM 1, 4 AND 6a-c RESPECTIVELY

\begin{tabular}{cllcccc}
\hline S. No. & \multicolumn{1}{c}{${ }^{a}$ Product } & \multicolumn{1}{c}{$\mathrm{R}_{1}$} & Time $(\mathrm{h})$ & Temp. $\left({ }^{\circ} \mathrm{C}\right)$ & ${ }^{\text {b}}$ Yield $(\%)$ & m.p. $\left({ }^{\circ} \mathrm{C}\right)$ \\
\hline 1 & $3 \mathrm{a}\left(\mathrm{Ar}=\mathrm{C}_{6} \mathrm{H}_{5}\right)$ & - & 1 & 100 & 75 & 205 \\
2 & $3 \mathrm{~b}\left(\mathrm{Ar}=\mathrm{C}_{6} \mathrm{H}_{4}-p-\mathrm{CH}_{3}\right)$ & - & 1 & 100 & 70 & $216-217$ \\
3 & $3 \mathrm{c}\left(\mathrm{Ar}=\mathrm{C}_{6} \mathrm{H}_{4}-p-\mathrm{Cl}\right)$ & - & 1 & 100 & 73 & 222 \\
4 & $7 \mathrm{a}\left(\mathrm{Ar}=\mathrm{C}_{6} \mathrm{H}_{5}\right)$ & $\mathrm{CH}_{3}$ & 3 & 160 & 70 & $114-115$ \\
5 & $7 \mathrm{~b}\left(\mathrm{Ar}=\mathrm{C}_{6} \mathrm{H}_{5}\right)$ & $\mathrm{C}_{2} \mathrm{H}_{5}$ & 3 & 170 & 68 & $158-160$ \\
6 & $7 \mathrm{c}\left(\mathrm{Ar}=\mathrm{C}_{6} \mathrm{H}_{5}\right)$ & $\mathrm{CH}_{2} \mathrm{Ph}$ & 3 & 170 & 70 & $120-121$ \\
7 & $7 \mathrm{~d}\left(\mathrm{Ar}=\mathrm{C}_{6} \mathrm{H}_{4}-p-\mathrm{CH}_{3}\right)$ & $\mathrm{CH}_{3}$ & 3 & 170 & 72 & $129-130$ \\
8 & $7 \mathrm{e}\left(\mathrm{Ar}=\mathrm{C}_{6} \mathrm{H}_{4}-p-\mathrm{CH}_{3}\right)$ & $\mathrm{C}_{2} \mathrm{H}_{5}$ & 3 & 170 & 70 & $108-110$ \\
9 & $7 \mathrm{f}\left(\mathrm{Ar}=\mathrm{C}_{6} \mathrm{H}_{4}-p-\mathrm{CH}_{3}\right)$ & $\mathrm{CH}_{2} \mathrm{Ph}$ & 3 & 180 & 65 & $210-212$ \\
10 & $7 \mathrm{~g}\left(\mathrm{Ar}=\mathrm{C}_{6} \mathrm{H}_{4}-p-\mathrm{Cl}\right)$ & $\mathrm{CH}_{3}$ & 3 & 175 & 73 & $143-145$ \\
11 & $7 \mathrm{~h}\left(\mathrm{Ar}=\mathrm{C}_{6} \mathrm{H}_{4}-p-\mathrm{Cl}\right)$ & $\mathrm{C}_{2} \mathrm{H}_{5}$ & 3 & 175 & 70 & $171-172$ \\
12 & $7 \mathrm{i}\left(\mathrm{Ar}=\mathrm{C}_{6} \mathrm{H}_{4}-p-\mathrm{Cl}\right)$ & $\mathrm{CH}_{2} \mathrm{Ph}$ & 3 & 180 & 72 & $>230$ \\
\hline
\end{tabular}

${ }^{a}$ All the products were characterized by ${ }^{1} \mathrm{H}$ NMR, IR and mass spectral data and comparison with the authentic samples available or prepared according to reported methods.

${ }^{\text {b} Y i e l d s ~ r e f e r s ~ t o ~ i s o l a t e d ~ y i e l d s . ~}$

$\left(\mathbf{5 b}, \mathrm{Ar}=\mathrm{C}_{6} \mathrm{H}_{4}-p-\mathrm{Cl}\right)$ and $\left(\mathbf{5 c}, \mathrm{Ar}=\mathrm{C}_{6} \mathrm{H}_{4}-p-\mathrm{CH}_{3}\right)$ in glycerol at 170-180 ${ }^{\circ} \mathrm{C}$ for $1 \mathrm{~h}$, resulted in 3a-c (i.e., 3a, $\left.\mathrm{Ar}=\mathrm{C}_{6} \mathrm{H}_{5}\right),(\mathbf{3 b}$, $\left.\mathrm{Ar}=\mathrm{C}_{6} \mathrm{H}_{4}-p-\mathrm{Cl}\right)$ and $\left(3 \mathbf{c}, \mathrm{Ar}=\mathrm{C}_{6} \mathrm{H}_{4}-p-\mathrm{CH}_{3}\right)$ in good yields (Table-1) also identical with the products obtained above in all respects (m.p. m.m.p. and co-TLC analysis).

The N-alkylation of (3a-c) and (4) and with alkylating agents such as dimethyl sulphate (DMS), diethyl sulphate (DES) and benzyl chloride $\left(\mathrm{PhCH}_{2} \mathrm{Cl}\right)$ in the presence of $\mathrm{K}_{2} \mathrm{CO}_{3}$ as a mild base and tetrabutylammonium bromide (TBAB) as phase transfer catalyst, by a simple physical grinding of the reactants in a mortar with a pestle under solvent-free conditions for $5 \mathrm{~min}$ at room temperature, gave respectively, $\mathrm{N}$-methyl2-styrylbenzimidazole (7a, i.e., $\mathrm{R}_{1}=\mathrm{CH}_{3}, \mathrm{Ar}=\mathrm{C}_{6} \mathrm{H}_{5}$ ), N-ethyl2-styrylbenzimidazole (7b, i.e., $\mathrm{R}_{1}=\mathrm{C}_{2} \mathrm{H}_{5}, \mathrm{Ar}=\mathrm{C}_{6} \mathrm{H}_{5}$ ), N-benzyl-2-styrylbenzimidazole (7c, i.e., $\mathrm{R}_{1}=\mathrm{PhCH}_{2} \mathrm{Cl}, \mathrm{Ar}=\mathrm{C}_{6} \mathrm{H}_{5}$ ), N-methyl-2-[(E)-2-(4-methylphenyl)ethenyl]-1H-1,3-benzimidazole (7d, i.e., $\left.\mathrm{R}_{1}=\mathrm{CH}_{3}, \mathrm{Ar}=\mathrm{C}_{6} \mathrm{H}_{4}-p-\mathrm{CH}_{3}\right)$, N-ethyl-2[(E)-2-(4-methylphenyl)ethenyl]-1H-1,3-benzimidazole (7e, i.e., $\mathrm{R}_{1}=\mathrm{C}_{2} \mathrm{H}_{5}$, $\left.\mathrm{Ar}=\mathrm{C}_{6} \mathrm{H}_{4}-p-\mathrm{CH}_{3}\right)$, N-benzyl-2-[(E)-2-(4methylphenyl)ethenyl]-1H-1, 3-benzimidazole (7f, i.e., $\mathrm{R}_{1}=$ $\left.\mathrm{PhCH}_{2} \mathrm{Cl}, \mathrm{Ar}=\mathrm{C}_{6} \mathrm{H}_{4}-p-\mathrm{CH}_{3}\right)$, N-methyl-2-[(E)-2-(4chlorophenyl)ethenyl]-1H-1,3- benzimidazole $\left(7 \mathrm{~g}\right.$, i.e., $\mathrm{R}_{1}=$ $\left.\mathrm{CH}_{3}, \mathrm{Ar}=\mathrm{C}_{6} \mathrm{H}_{4}-p-\mathrm{Cl}\right)$, N-ethyl-2-[(E)-2-(4-chlorophenyl) ethenyl]-1H-1, 3-benzimidazole (7h, i.e., $\mathrm{R}_{1}=\mathrm{C}_{2} \mathrm{H}_{5}, \mathrm{Ar}=\mathrm{C}_{6} \mathrm{H}_{4}$ $p$-Cl) and N-benzyl-2-[(E)-2-(4-chlorophenyl)ethenyl]-1H1,3-benzimidazole (7i, i.e., $\mathrm{R}_{1}=\mathrm{PhCH}_{2} \mathrm{Cl}, \mathrm{Ar}=\mathrm{C}_{6} \mathrm{H}_{4}-p$-Cl) respectively (Table-2).

Compound $\mathbf{4}$ on treatment with alkylating agents in the presence of $\mathrm{K}_{2} \mathrm{CO}_{3}$ and TBAB under physical grinding conditions in a mortar with pestle gave the products N-methyl-2methylbenzimidazole $\left(\mathbf{6 a}\right.$, i.e., $\left.\mathrm{R}=\mathrm{CH}_{3}\right)$, N-ehtyl-2methylbenzimidazole (6b, i.e., $\left.\mathrm{R}=\mathrm{C}_{2} \mathrm{H}_{5}\right)$ and N-benzyl-2mehtylbenzimidazole $\left(\mathbf{6 c}\right.$, i.e., $\mathrm{R}=\mathrm{PhCH}_{2} \mathrm{Cl}$ ) respectively and these products were identical with the ones prepared earlier ${ }^{22,25}$ using conventional methods in all respects (m.p. m.m.p and co-TLC analysis) (Table-2).

The $\mathrm{N}$-alkylation reactions of $\mathbf{3 a - c}$ and $\mathbf{4}$ were also carried out in PEG-600 as a solvent, both by heating at $100{ }^{\circ} \mathrm{C}$ on a water bath and in microwave method. Thus the treatment of 3a-c and 4, independently, with each of dimethyl sulphate (DMS), diethyl sulphate (DES) and benzyl chloride $\left(\mathrm{PhCH}_{2} \mathrm{Cl}\right)$

\begin{tabular}{|c|c|c|c|c|c|c|}
\hline \multicolumn{7}{|c|}{$\begin{array}{c}\text { TABLE-2 } \\
\text { N-ALKYLARALKYLATIONS OF COMPOUNDS } \\
\text { 3a-c AND } 4 \text { USING SOLID PHASE SYNTHESIS } \\
\text { (BY SIMPLE PHYSICAL GRINDING) }\end{array}$} \\
\hline \multirow[t]{2}{*}{$\begin{array}{l}\text { S. } \\
\text { No. }\end{array}$} & \multirow[t]{2}{*}{ Substrate } & \multirow[t]{2}{*}{ Reagent } & \multirow[t]{2}{*}{ Product $^{\mathrm{a}}$} & \multicolumn{3}{|c|}{$\begin{array}{l}\text { Solid-phase (simple } \\
\text { physical grinding) }\end{array}$} \\
\hline & & & & $\begin{array}{l}\text { Time } \\
(\mathrm{min})\end{array}$ & $\begin{array}{l}\text { Temp. } \\
\left({ }^{\circ} \mathrm{C}\right)\end{array}$ & $\begin{array}{c}\text { Yield }^{\mathrm{b}} \\
(\%)\end{array}$ \\
\hline \multirow[t]{3}{*}{1} & $3 \mathbf{a}$ & DMS & $7 a$ & 5 & RT & 80 \\
\hline & $\left(\mathrm{R}=\mathrm{CH}_{3}\right)$ & DES & $7 b$ & 5 & RT & 78 \\
\hline & & $\mathrm{Ph}-\mathrm{CH}_{2} \mathrm{Cl}$ & $7 c$ & 10 & RT & 90 \\
\hline \multirow[t]{3}{*}{2} & $3 b$ & DMS & 7d & 5 & RT & 80 \\
\hline & $(\mathrm{R}=\mathrm{OH})$ & DES & $7 e$ & 8 & RT & 75 \\
\hline & & $\mathrm{Ph}-\mathrm{CH}_{2} \mathrm{Cl}$ & $7 f$ & 10 & RT & 78 \\
\hline \multirow[t]{3}{*}{3} & $3 c$ & DMS & $7 g$ & 6 & RT & 90 \\
\hline & $(\mathrm{R}=\mathrm{Cl})$ & DES & $7 \mathrm{~h}$ & 10 & RT & 80 \\
\hline & & $\mathrm{Ph}-\mathrm{CH}_{2} \mathrm{Cl}$ & $7 \mathbf{i}$ & 10 & RT & 78 \\
\hline \multirow[t]{3}{*}{4} & 4 & DMS & $6 a$ & 5 & RT & 80 \\
\hline & & DES & $6 b$ & 10 & RT & 78 \\
\hline & & $\mathrm{PhCH}_{2} \mathrm{Cl}$ & $6 c$ & 10 & RT & 80 \\
\hline
\end{tabular}

${ }^{a}$ All the products were characterized by ${ }^{1} \mathrm{H}$ NMR, IR and mass spectral data and comparison with the authentic samples available or prepared according to reported methods.

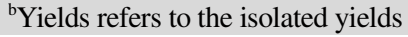

in PEG-600 at $100{ }^{\circ} \mathrm{C}$ about $1 \mathrm{~h}$ without the use of any base, followed by simple processing, gave respectively, 7a-i and 6a-c (Table-3).

Compounds $7 \mathbf{a}-\mathbf{i})$ and $\mathbf{6 a - c}$ were also be prepared by an alternative method. Thus, $\mathbf{3 a - c}$ and $\mathbf{4}$ on reactions, independently, with each of dimethyl sulphate (DMS), diethyl sulphate (DES) and benzyl chloride $\left(\mathrm{PhCH}_{2} \mathrm{Cl}\right)$ under microwave irradiation conditions for 5-10 $\mathrm{min}$ and subsequent processing, gave 7a-i and 6a-c (Table-4).

\section{Conclusion}

In conclusion, green and simple syntheses of 2styrylbenzimidazoles (3a-c) and their $\mathrm{N}$-alkyl/aralkyl derivatives 7a-i were described. It appears from this study that green syntheses using solvents such as glycerol and PEG-600 and eco-friendly methods like solid phase synthesis (physical grinding) and microwave irradiation gives better yields, quality and in less reaction time the products over conventional methods. The entire sequence of reactions shown in Scheme-I has been carried out using eco-friendly solvents and green conditions. 


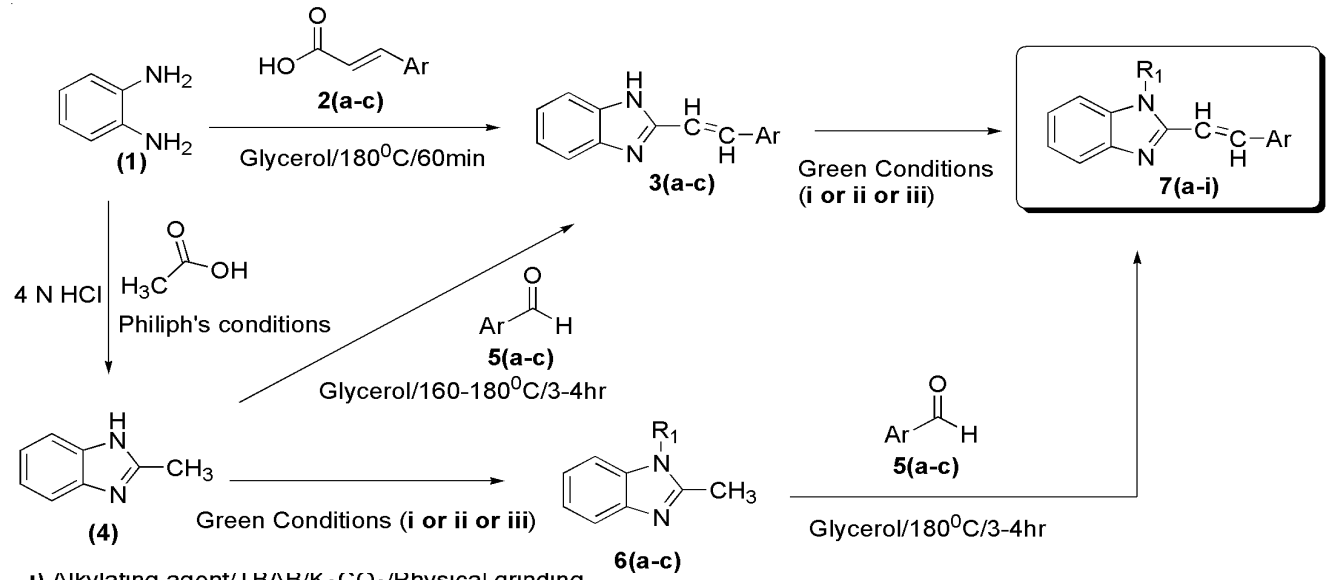

1) Alkylatıng agent/ I $\mathrm{BAB} / \mathrm{K}_{2} \mathrm{CO}_{3} / \mathrm{Phys}$ sical grındıng ii) Alkylating agent $/ P E G-600 / 100^{\circ} \mathrm{C} / 60 \mathrm{~min}$ iii) Alkylating agent/PEG-600/MW/450 W/5min

Where, Ar $=\mathrm{C}_{6} \mathrm{H}_{5},-p-\mathrm{Cl}-\mathrm{C}_{6} \mathrm{H}_{4},-p-\mathrm{CH}_{3}-\mathrm{C}_{6} \mathrm{H}_{4}$ $\mathbf{R}_{1}=-\mathrm{CH}_{3},-\mathrm{CH}_{2}-\mathrm{CH}_{3},-\mathrm{CH}_{2}-\mathrm{Ph}$

Scheme-I: Synthesis \& N-alkylation studies of 2-styrylbenzimidazoles 7a-i

\begin{tabular}{|c|c|c|c|c|c|c|}
\hline 3a-c & $\begin{array}{r}N \text {-ALKYI } \\
\text { ND } 4 \text { USIN }\end{array}$ & $\begin{array}{r}\mathrm{T} \\
\text { ARALKYL } \\
\mathrm{J} \text { PEG-600 }\end{array}$ & $\begin{array}{l}\text { BLE-3 } \\
\text { TIONS OI } \\
\text { S SOLVEI }\end{array}$ & $\begin{array}{l}\text { COMP } \\
\text { T (SOL }\end{array}$ & $\begin{array}{l}\text { UNDS } \\
\text { TION P }\end{array}$ & IASE) \\
\hline \multirow[t]{2}{*}{ S.No } & \multirow[t]{2}{*}{ Substrate } & \multirow[t]{2}{*}{ Reagent } & \multirow[t]{2}{*}{ Product $^{\mathrm{a}}$} & \multicolumn{3}{|c|}{$\begin{array}{l}\text { Green solvent (solution } \\
\text { phase) in PEG-600 }\end{array}$} \\
\hline & & & & $\begin{array}{l}\text { Time } \\
(\mathrm{min})\end{array}$ & $\begin{array}{c}\text { Temp } \\
\left.{ }^{\circ} \mathrm{C}\right)\end{array}$ & $\begin{array}{c}\text { Yield }^{\mathrm{b}} \\
(\%)\end{array}$ \\
\hline \multirow[t]{3}{*}{1.} & $3 \mathbf{a}$ & DMS & $7 \mathbf{a}$ & 60 & 100 & 82 \\
\hline & $\left(\mathrm{R}=\mathrm{CH}_{3}\right)$ & DES & $7 b$ & 80 & 100 & 75 \\
\hline & & $\mathrm{PhCH}_{2} \mathrm{Cl}$ & 7c & 130 & 100 & 72 \\
\hline \multirow[t]{3}{*}{2.} & $3 \mathbf{b}$ & DMS & 7d & 60 & 100 & 80 \\
\hline & $(\mathrm{R}=\mathrm{OH})$ & DES & $7 e$ & 60 & 100 & 70 \\
\hline & & $\mathrm{PhCH}_{2} \mathrm{Cl}$ & $7 f$ & 130 & 100 & 75 \\
\hline \multirow[t]{3}{*}{3.} & $3 c$ & DMS & $7 \mathrm{~g}$ & 60 & 100 & 83 \\
\hline & $(\mathrm{R}=\mathrm{Cl})$ & DES & $7 \mathrm{~h}$ & 60 & 100 & 76 \\
\hline & & $\mathrm{PhCH}_{2} \mathrm{Cl}$ & $7 \mathbf{i}$ & 120 & 100 & 78 \\
\hline \multirow{2}{*}{4.} & & DES & $6 b$ & 80 & 100 & 75 \\
\hline & 4 & $\mathrm{PhCH}_{2} \mathrm{Cl}$ & $6 c$ & 120 & 100 & 68 \\
\hline
\end{tabular}

\begin{tabular}{|c|c|c|c|c|c|c|}
\hline & $N$-ALK & $\begin{array}{l}\text { /ARALK } \\
\text {-c AND } 4\end{array}$ & $\begin{array}{l}\text { LABLE-4 } \\
\text { LATIONS } \\
\text { SING MI }\end{array}$ & $\begin{array}{l}\text { DF CON } \\
\text { ROWA }\end{array}$ & $\begin{array}{l}\text { POUNDS } \\
\mathrm{E}\end{array}$ & \\
\hline S.No & Substrate & Reagent & Product $^{\mathrm{a}}$ & Micr & wave irra & ation \\
\hline & & & & $\begin{array}{l}\text { Time } \\
(\mathrm{min})\end{array}$ & $\begin{array}{l}\text { Temp } \\
\left.{ }^{(0} \mathrm{C}\right) / \\
\text { Watt }\end{array}$ & $\begin{array}{c}\text { Yield }^{\mathrm{b}} \\
(\%)\end{array}$ \\
\hline & & DMS & $7 a$ & 3 & $100 / 450$ & 88 \\
\hline 1. & & DES & $7 b$ & 5 & $100 / 450$ & 80 \\
\hline & $\left(\mathrm{R}=\mathrm{CH}_{3}\right)$ & $\mathrm{PhCH}_{2} \mathrm{Cl}$ & $7 c$ & 5 & $100 / 450$ & 75 \\
\hline 2. & $\mathbf{3 b}$ & DMS & 7d & 5 & $100 / 450$ & 80 \\
\hline & $(\mathrm{R}=\mathrm{OH})$ & DES & $7 e$ & 5 & $100 / 450$ & 78 \\
\hline & & $\mathrm{PhCH}_{2} \mathrm{Cl}$ & $7 f$ & 5 & $100 / 450$ & 75 \\
\hline 3. & & DMS & $7 g$ & 5 & $100 / 450$ & 88 \\
\hline & $(\mathrm{R}=\mathrm{Cl})$ & DES & $7 \mathrm{~h}$ & 5 & $100 / 450$ & 78 \\
\hline & & $\mathrm{PhCH}_{2} \mathrm{Cl}$ & $7 \mathbf{i}$ & 5 & $100 / 450$ & 75 \\
\hline 4 & 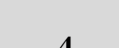 & DES & $6 b$ & 5 & $100 / 450$ & 85 \\
\hline 4. & 4 & $\mathrm{PhCH}_{2} \mathrm{Cl}$ & $6 c$ & 5 & $100 / 450$ & 82 \\
\hline
\end{tabular}

${ }^{a}$ All the products were characterized by ${ }^{1} \mathrm{H}-\mathrm{NMR}$, IR and Mass spectral data and comparison with the authentic samples available or prepared according to reported methods.

${ }^{\text {b}}$ Yields refers to the isolated yields

\section{ACKNOWLEDGEMENTS}

The authors are thankful to the authorities of Jawaharlal Nehru Technological University Hyderabad, for providing financial support and laboratory facilities.

\section{REFERENCES}

1. Y. Gu, J. Barrault and F. Jerome, Adv. Synth. Catal., 350, 2007 (2008).

2. Y. Gu and F. Jerome, Green Chem., 12, 1127 (2010).

3. A. Wolfson, C. Dlugy and Y. Shotland, Environ. Chem. Lett., 5, 67 (2007).

4. D.T. Nannapaneni, A.V.S.S.S. Gupta, M.I. Reddy, and R.C. Sarva, J. Young Pharm., 2, 273 (2010).

5. D.A. Horton, G.T. Bourne and M.L. Smythe, Chem. Rev., 103, 893 (2003).

6. A.R. Porcari, R.V. Devivar, L.S. Kucera, J.C. Drach and L.B. Townsend, J. Med. Chem., 41, 1252 (1998).

7. M.T. Migawa, J.L. Girardet, J.A. Walker, G.W. Koszalka, S.D. Chamberlain, J.C. Drach and L.B. Townsend, J. Med. Chem., 41, 1242 (1998).

8. I. Tamm, Science, 126, 1235 (1957).

9. P. Tempest, V. Ma, S. Thomas, Z. Hua, M.G. Kelly and C. Hulme, Tetrahedron Lett., 42, 4959 (2001).

10. M.M. Heravi, S. Sadjadi, H.A. Oskooie, R.H. Shoar and F.F. Bamoharram, Catal. Commun., 9, 504 (2008).

11. K. Bahrami, M. Khodaei and I. Kavianinia, Synthesis, 547 (2007).

12. H. Xiangming, M. Huiqiang and W. Yulu, ARKIVOC, 150 (2007).

13. B. Das, H. Holla and Y. Srinivas, Tetrahedron Lett., 48, 61 (2007).

14. Y. Wang, K. Sarris, D.R. Sauer and S.W. Djuric, Tetrahedron Lett., 47, 4823 (2006).

15. Y. Wang, H. Ma, X. Han and J. Wang, Heterocycles, 71, 1821 (2007).

16. Y. Tagawa, K. Yamagata and K. Sumoto, Heterocycles, 75, 415 (2008).

17. R. Varala, A. Nasreen, R. Enugala and S.R. Adapa, Tetrahedron Lett., 48, 69 (2007)

18. P.K. John, L. Jesper and C.F.J. Raymond, Tetrahedron Lett., 41, 5410 (2000).

19. V. Ravi, E. Ramu, K. Vijay and A.S. Rao, Chem. Pharm. Bull. (Tokyo), 55, 1254 (2007).

20. A. Chawla, R. Kaur and A. Goyal, J. Chem. Pharm. Res., 3, 925 (2011).

21. P.K. Dubey, D.E. Rao, J. Ramanatham, R. Kumar and J.S. Grossert, J. Indian Chem. Soc., 75, 460 (1998).

22. P.K. Dubey, R. Kumar, J.S. Grossert and D.L. Hooper, Indian J. Chem., 37B, 288 (1998).

23. P.K. Dubey and R. Kumar, Indian J. Chem., 38B, 1041 (1999).

24. K. Ramaiah, P.K. Dubey, D.E. Rao, J. Ramanatham and R. Kumar, Indian J. Chem., 39B, 904 (2000).

25. P.K. Dubey and R. Kumar, Indian J. Heterocycl. Chem., 16, 131 (2006).

26. M.A. Phillips, J. Chem. Soc., 928, 2393 (1928).

27. K. Selvam and M. Swaminathan, Tetrahedron Lett., 52, 3386 (2011). 